\title{
TRATAMENTO ENDOVASCULAR DE DISSECÇÃO CRÔNICA TORACOABDOMINAL COMPLICADA COM ANEURISMA TORÁCICO, MEDIANTE IMPLANTE DE ENDOPRÓTESE VASCULAR
}

\author{
ENDOVASCULAR TREATMENT FOR CHRONIC TORACHO-ABDOMINAL \\ AORTIC DISSECTION COMPLICATED WITH THORACIC ANEURYSM, BY THE \\ PLACEMENT OF AN ENDOVASCULAR STENT-GRAFT
}

\author{
Gaudencio Espinosa, TCBC-RJ ${ }^{1}$ \\ Jesus Antonio Carvalho Abreu ${ }^{2}$ \\ Adalberto Pereira Araujo ${ }^{3}$
}

\section{INTRODUÇÃO}

A mortalidade cirúrgica da dissecção crônica da aorta torácica supera de forma significativa a do tratamento clínico, no entanto as dissecções crônicas da aorta torácica descendente podem, ao longo do tempo, evoluir para aneurismas. ${ }^{1}$

O tratamento tradicional para pacientes com aneurisma de aorta torácica descendente consiste na intervenção cirúrgica com interposição de enxerto vascular. Estas cirurgias representam um grande trauma, com substancial morbidade e mortalidade em um grupo de pacientes freqüentemente idosos e debilitados por outros problemas médicos. ${ }^{1}$

O implante de endopróteses vasculares para o tratamento das doenças que acometem a aorta foi introduzido por Parodi em 1990, representando uma alternativa inovadora e menos agressiva do que os procedimentos cirúrgicos convencionais. ${ }^{1,2}$

Neste trabalho, o nosso objetivo é relatar o caso de um paciente com dissecção crônica toracoabdominal associada à aneurisma de aorta torácica descendente, em expansão, e com sintomatologia dolorosa, tratado de forma efetiva mediante o implante de uma endoprótese vascular.

\section{RELATO DO CASO}

Paciente de 68 anos, sexo masculino, branco, hipertenso e coronariopata, com relato de infarto do mio- cárdio há dez anos. Apresentava dissecção de aorta toracoabdominal tipo B de Daily e Miller ou tipo IIIb de DeBakey, diagnosticada há dois anos e acompanhada em outro hospital através de tomografia computadorizada (TC) semestral.

Procurou o serviço de cirurgia vascular do Hospital Clementino Fraga Filho (UFRJ) em junho/99 com queixa de dor abdominal intensa, irradiando para o dorso e de difícil controle, apesar da administração de morfínicos. Foi realizada TC de emergência que evidenciou aneurisma de aorta torácica descendente e extensão da dissecção aortica até as artérias ilíacas (Figura 1A).

A dilatação aneurismática da aorta torácica descendente (tipo I de Crawford), apresentava 69mm no seu maior diâmetro, associando-se à dissecção toracoabdominal que se iniciava $3 \mathrm{~cm}$ distalmente à origem da artéria subclávia esquerda e se estendia até as artérias ilíacas, englobando a origem dos vasos viscerais (Figua 1B) e a bifurcação aórtica. Observou-se ainda, orifício de 'entrada' do falso lúmen na transição do terço superior para o médio da artéria torácica descendente. Ao exame físico apresentava pressão arterial de 150x100mmHg; abdome flácido, levemente doloroso à palpação, e com boa peristalse; pulsos amplos em artérias femorais, poplíteas, tibiais posteriores e pediosas.

Ecocardiograma evidenciando ectasia de aorta ascendente ao nível dos seios coronarianos; ao doppler apresentava fluxo retrógrado mitral compatível com déficit de relaxamento de ventrículo esquerdo e regurgitação aórtica mínima. Fração de ejeção de 52\%.

1. Professor Adjunto de Cirurgia da UFRJ

2. Médico Residente de Cirurgia Vascular do HU-HFRJ

3. Professor convidado da UFRJ.

Recebido em 12/11/99

Aceito para publicação em 14/3/2000

Trabalho realizado no Serviço de Cirurgia Vascular do Hospital Universitário Clementino Fraga Filho (HU-UFRJ) e no Departamento de Cirurgia da Universidade Federal do Rio de Janeiro (UFRJ). 
O procedimento endovascular foi realizado sob anestesia geral. Inicialmente o paciente foi submetido à punção e à cateterização pela técnica de Seldinger da artéria

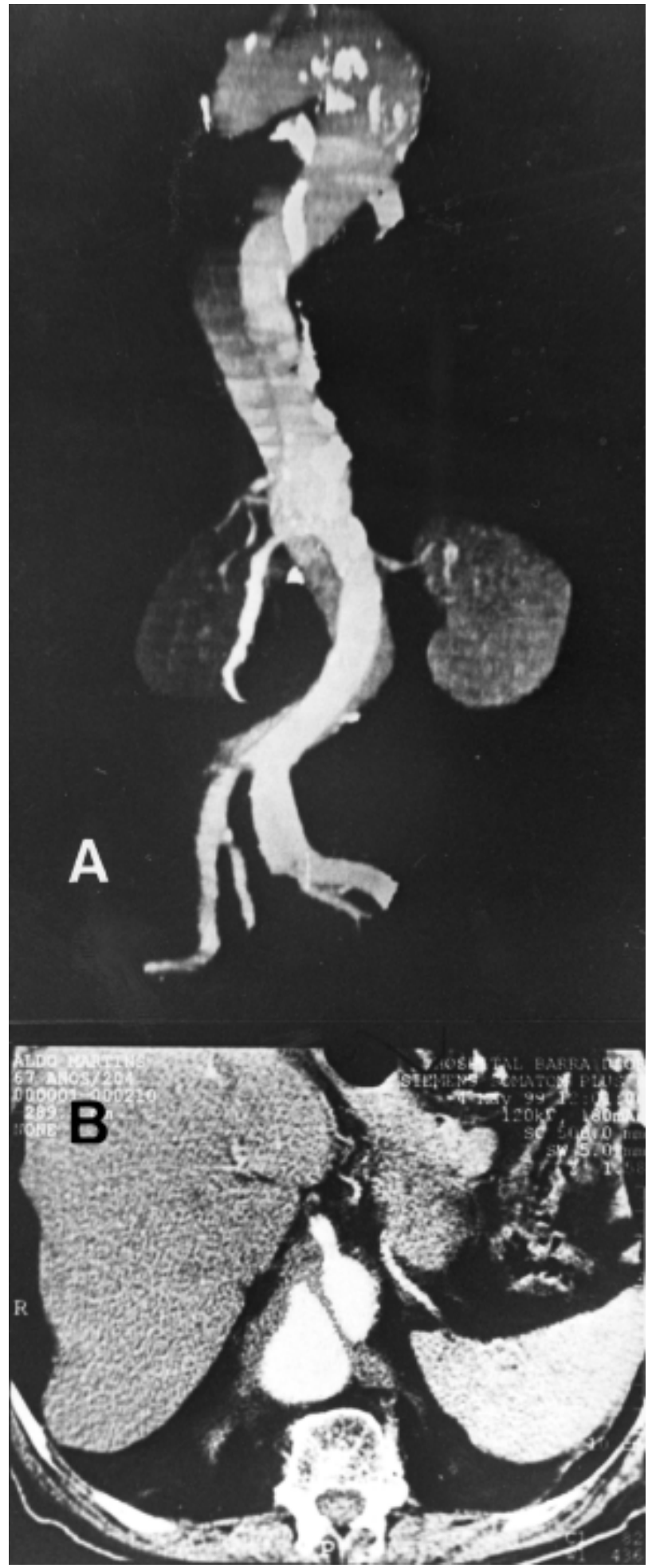

Figura 1 - Tomografia helicoidal pré-operatória. (A) Reconstrução tridimensional evidenciando linha de dissecção acometendo toda a extensão da aorta toracoabdominal. (B) No corte axial ao nível do tronco celíaco, evidencia-se duplo lúmen aórtico. braquial esquerda, introduzindo um cateter tipo pigtail de 5 french o qual foi posicionado na aorta torácica para realizar os controles angiográficos durante o procedimento.

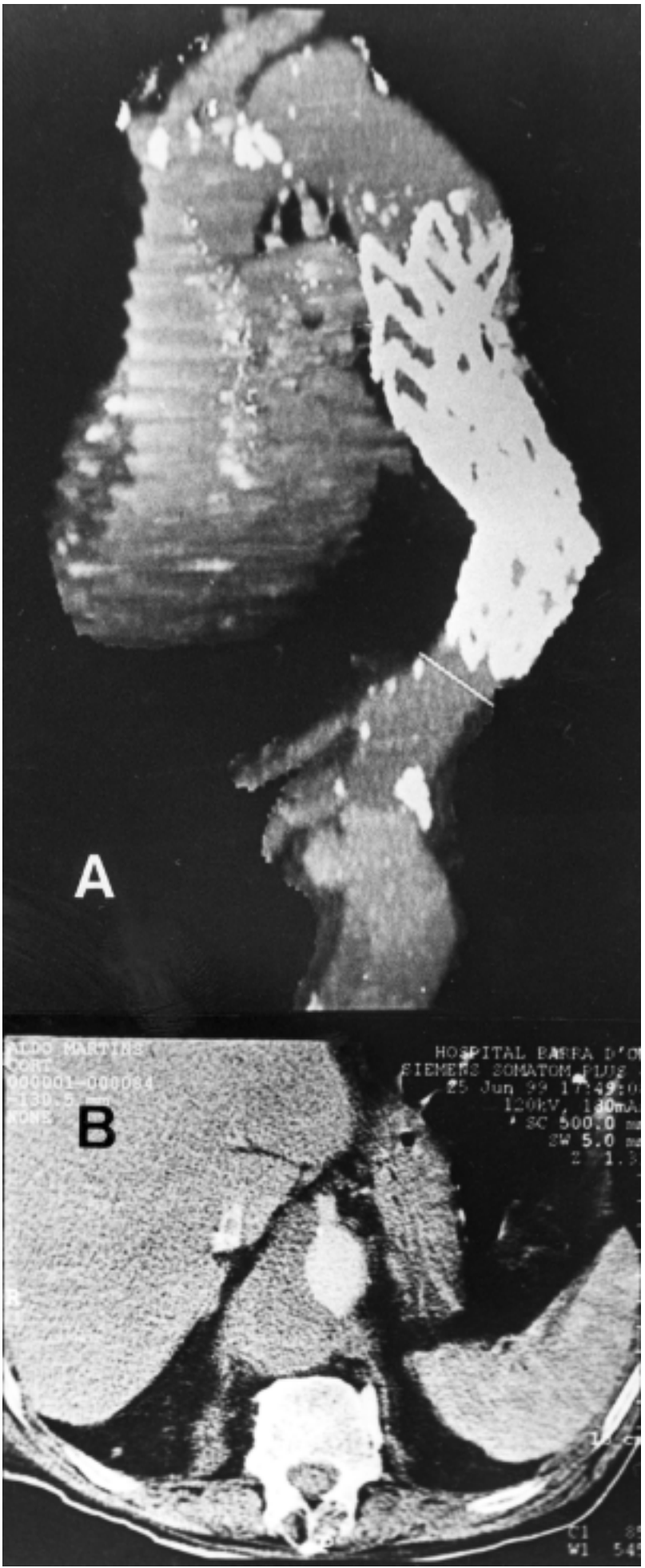

Figura 2 - Tomografia helicoidal pós-operatória. (A) Reconstrução tridimensional demonstrando o bom posicionamento da endoprótese vascular. (B) No corte axial no nível do tronco celíaco, evidencia-se trombose do falso lúmen aórtico. 
Posteriormente foi realizada dissecção cirúrgica e reparo da artéria femoral comum esquerda, com introdução, em seguida, de cateter tipo headhunter, de 5 french, no sentido proximal de diâmetro, e posterior posicionamento de fio guia de troca super-rígido de $0,035 "$ ". A arteriografia transoperatória demonstrou que ambos os cateteres se localizavam no lúmen aórtico verdadeiro. Após arteriotomia longitudinal, o sistema liberador da endoprótese vascular (24 french) foi introduzido pela artéria femoral, apoiado sobre o guia super-rígido, e avançado sob controle radioscópico em direção à aorta torácica descendente.

Após a redução da pressão arterial média para $60 \mathrm{mmHg}$ com vasodilatadores endovenosos, realizamos sem intercorrências o implante de uma endoprótese vascular tipo TALENT ${ }^{\circledR}$. Esta foi fabricada sob medida, para adequar-se à aorta do paciente, sendo de $46 \mathrm{~mm}$ no seu diâmetro proximal, de $34 \mathrm{~mm}$ de diâmetro distal e $15 \mathrm{~cm}$ de longitude recoberta. A endoprótese possuía ainda o seu primeiro anel metálico livre de tecido totalizando $16,5 \mathrm{~cm}$ de extensão.

A endoprótese foi implantada de forma adequada, tangenciando o óstio da artéria subclávia esquerda. No estudo angiográfico de controle evidenciamos oclusão do orifício de 'entrada' da dissecção, encontrando-se a endoprótese de forma hermética tanto na sua fixação proximal como distal.

O procedimento foi concluído ao cabo de três horas, sem intercorrências. Não houve necessidade de transfusão sangüínea. O paciente recebeu alta do CTI no primeiro dia de pós-operatório e do hospital no sexto dia, sem que ocorressem complicações importantes.

Após uma semana, foi realizada TC helicoidal com contraste que revelou bom posicionamento da endoprótese (Figura 2A), com exclusão do aneurisma e correção do falso lúmen (Figura $2 \mathrm{~B}$ ). No controle tomográfico do paciente, após seis meses da realização do procedimento, observamos uma diminuição do diâmetro do aneurisma para $56 \mathrm{~mm}$

\section{DISCUSSÃO}

Os aneurismas toracoabdominais têm risco de ruptura significativamente superior aos infra-renais, variando de $46 \%$ a $74 \%$, segundo diversos relatos. ${ }^{1}$ A sobrevida em cinco anos é muito baixa, entre $9 \%$ e $13 \%,{ }^{2}$ sendo mandatória a consideração de tratamento cirúrgico em todos os indivíduos que são portadores desta doença.

O tratamento cirúrgico convencional apresenta um índice de mortalidade de $10 \%$ a $20 \%$, e $10 \%$ de paraplegia, aumentando para $50 \%$ e $30 \%$ respectivamente, em situações de emergência..$^{1,3,4}$ Uma alta taxa de morbidade ocorre em conseqüência do sangramento transoperatório, das repercussões do aumento de pós-carga, do tempo de isquemia das vísceras e das alterações do equilíbrio ácido-básico e hidreletrolíltico. ${ }^{5}$

Com o avanço das técnicas de cirurgia endovascular, o implante de endopróteses em aorta torácica surge como importante alternativa à cirurgia convencional em casos selecionados, de modo a possibilitar um procedimento menos agressivo, com taxa de mortalidade de $9 \%$ e paraplegia de 3\%, com uma menor necessidade de transfusão sangüínea e redução considerável do tempo de internação hospitalar. ${ }^{1}$

No caso relatado neste trabalho o paciente apresentava um grande risco clínico para a cirurgia convencional, devido à coronariopatia grave e ao infarto prévio, com fração de ejeção significativamente diminuída. Além disso, apresentava uma evidente dificuldade técnica para a cirurgia, devida à grande extensão da dissecção, a qual se iniciava na aorta torácica e se estendia até as artérias ilíacas. Desta forma o paciente foi considerado como candidato adequado para tratamento endovascular.

O diâmetro da endoprótese deve exceder $10 \%$ a $15 \%$ o diâmetro do 'colo' da aorta medido pela TC, para prover uma força radial residual e uma efetiva ancoragem após a implantação do stent. ${ }^{1,3} \mathrm{O}$ ideal é que os 'colos' aórticos (ou zonas de ancoragem) proximal e distal sejam mais longos que $20 \mathrm{~mm}$ e cilíndricos. ${ }^{1,3}$ No caso relatado havia dissecção associada ao aneurisma, a qual se iniciava a $3 \mathrm{~cm}$ da emergência da artéria subclávia esquerda, favorecendo uma boa ancoragem proximal. Apesar de apresentar uma grande tortuosidade em seu trajeto proximal, não houve nenhuma dificuldade técnica.

$\mathrm{Na}$ cirurgia convencional o risco de paraplegia pode variar de 10 a $30 \%, 1,3,4$ sendo significativamente menor na técnica endovascular, em torno de $3 \% .{ }^{1}$ Esta temida complicação parece estar relacionada com o tempo de oclusão do fluxo sangüíneo na aorta, bem como com o tempo de hipotensão, geralmente causada por hipovolemia. No caso apresentado, não houve necessidade de ocluir o fluxo sangüíneo aórtico. Também não ocorreram perdas volêmicas, não havendo necessidade de transfusões sangüíneas, mantendo-se o paciente com boa estabilidade hemodinâmica durante todo o procedimento.

O paciente evoluiu de forma satisfatória no pós-operatório, recebendo alta do CTI após 24 horas do procedimento e do hospital no sexto dia, sem que ocorressem complicações importantes. Desta forma, a técnica endovascular neste paciente também contribuiu para um curto período de internação hospitalar.

\begin{abstract}
Our objective is to report a case of a patient with a descending thoracic aortic aneurysm and chronic aortic dissection, who was submitted to an endovascular treatment. A 68-year-old male with coronary artery disease and hypertension, with no history of trauma, diabetes or smoking. He had myocardial infarction ten years ago. Under general anesthesia, the left femoral artery was surgically exposed and the left braquial artery was catheterized with a "pigtail" catheter, under Seldinger technique. The proximal $46 \mathrm{~mm} / \varnothing$ and distal $34 \mathrm{~mm} / \varnothing$ stent-
\end{abstract}


graft was placed just distal to the origen of the left subclavian artery. Control arteriography showed that the lesion was completely excluded. The patient was discharged seven days after the surgery, when a computed tomographic control, was performed showing a sustained aneurysm exclusion and a satisfactory endovascular position.

Key Words: Aneurysm; Descending toracic aorta; Chronic aortic dissection; Endovascular; stent-graft.

\section{REFERÊNCIAS}

1. Dake MD, Miller DC, Mitchell RS, at al: The "first generation" of endovascular stent-grafts for patients with aneurysms of the descending thoracic aorta. J Thoracic Cardiovasc Surg 1998;116:689-704.

2. Nienaber CA, Fattori R, Lund G, at al: Nonsurgical reconstruction of thoracic aortic dissection by stent-graft placement. N Engl J Med 1999;340:1539-1545.

3. Rousseau H, Soula P, Perreault P, at al: Delayed treatment of traumatic rupture of the thoracic aorta with endoluminal covered stent. Circulation 1999;99:498-504.

4. Dake MD, Kato N, Mitchell RS, at al: Endovascular stentgraft placement for the treatment of acute aortic dissection. N Engl J Med 1999;340:1546-1552.
5. Vlahakes GJ: Catheter-based treatment of aortic dissection. N Engl J Med 1999;340:1585-1586.

\section{ENDEREÇO PARA CORRESPONDÊNCIA}

Dr. Gaudencio Espinosa

Praia de Icaraí, 113/801

24.230-001 - Niterói-RJ 